\title{
COMPUTER INFORMATION-AND-MEASURING SYSTEM FOR INVESTIGATION OF ARC SURFACING PROCESSES
}

\author{
I.A. RYABTSEV, Yu.N. LANKIN, V.G. SOLOVIOV, \\ P.P. OSECHKOV, V.A. TISHCHENKO and A.G. TIKHOMIROV \\ E.O. Paton Electric Welding Institute, NASU \\ 11 Bozhenko Str., 03680, Kiev, Ukraine. E-mail: office@paton.kiev.ua
}

\begin{abstract}
On the basis of portable computer and advanced software the information-and-measuring system was developed, which is intended to investigate the peculiarities and next automation of the arc surfacing processes. In the surfacing installation equipped with the developed computer information-and-measuring system (CIMS) it is possible to carry out experimental investigation of the influence of surfacing method, diameter or cross section of the used electrode material on its welding and technological characteristics and quality of the deposited beads and layers formation with a high degree of accuracy and reliability. CIMS with accumulation of databases on technologies of arc surfacing of different parts can serve as a basis for creation of systems of automatic control of arc surfacing processes. 3 Ref., 1 Table, 4 Figures.
\end{abstract}

$\boldsymbol{K} \boldsymbol{e} \boldsymbol{y} \boldsymbol{w} \boldsymbol{o} \boldsymbol{r} \boldsymbol{d} \boldsymbol{s}:$ arc surfacing, arc surfacing automation, flux-cored wires, surfacing modes, surfacing technologies

Due to its versatility the arc surfacing is used for restoration and strengthening of the parts of different configurations, sizes and mass. At the same time, during surfacing of parts (sheets) having a small thickness (less than $5 \mathrm{~mm}$ ), because of the risk of burns-through it is necessary to use electrode wires of small diameters, high deposition rate and the lowest possible surfacing performance modes in current and voltage. However, it can reduce the stability and efficiency of surfacing process and deteriorate the formation of deposited beads. At the same time, if there is no danger of burns-through, then for improving the efficiency the maximum surfacing modes are used. As a result the penetration of base metal and its volume in the deposited metal are increased, which often leads to the need in performing the multi-layer surfacing.

In practice, in the production the solution of the problem of development of the technology and techniques for surfacing the specific part depends on the experience of a surfacing technologist which does not always leads to reaching the optimum result. To avoid the possible errors in development of surfacing technologies, a higher degree of automation and computerization of the development and control of arc surfacing processes is required.

The development of systems of automatic control of processes of arc surfacing of different parts requires consideration of many parameters. It should provide the possibility of selection of the type of electrode material (solid or flux-cored wire, cold-rolled or flux-cored strip) and its sizes (diameter, cross section); surfacing method (submerged, open-arc or in shielding gases); presetting and maintaining the preset conditions and techniques of surfacing; marking of accidental or intentional deviations from the preset surfacing modes; memorization and subsequent use of optimum modes of surfacing the specific parts. The existing experience [1-3] shows that the modern level of development of computer science allows solving this problem.

If not to complicate the problem of development of automatic control systems for arc surfacing process by selection of chemical composition of the deposited metal, produced on the basis of the service conditions of the specific deposited part, then the approximate list of data necessary for development of such systems has the following form: surfacing voltage $U_{\mathrm{s}}(t)$; surfacing current $I_{\mathrm{s}}(t)$; diameter (cross section) of the electrode material; feed speed of the electrode material; deposition rate; surfacing heat input; stickout of electrode wire (strip); geometric dimensions of deposited beads and overlapping of adjacent deposited beads; geometric dimensions of part and specimen being deposited; grade of welding wire (strip); grade of base metal; need in shielding gas and its composition; need in flux and its grade; need in preheating and post surfacing heat treatment; integrated indicator of quality of surfacing, taking into account the geometric dimensions and deposited metal formation, volume of base metal in the deposited metal, surfacing efficiency, character of molten electrode metal transfer and process running stability, etc. 
The existing universal surfacing installations of type UMN-4, UMN-10, U-653, etc., as well as surfacing machines (installations) of type AD-231, A-1406, A-1412, are generally completed with the power sources of type VDU-506, VDU-1201, or VDU-1202, which are designed for surfacing currents of up to 500 and $1200 \mathrm{~A}$, respectively. In this equipment the setting up of surfacing conditions as to voltage is produced using potentiometers in the control circuit of the power source and installation, and as to current it is made using the potentiometer of regulating the feed speed of the electrode wire in the control circuit of surfacing installation. Taking this fact into account, the setting up of necessary surfacing conditions as to current and voltage are performed by changing the position of the potentiometer for voltage adjustment $P_{u}$ and the position of the potentiometer for adjustment of wire feed speed $P_{v p}$ during surfacing of reference specimens.

For automatic determination of the potentiometers position $\left(P_{u}\right.$ and $\left.P_{v p}\right)$ in accordance with the preset mean values of surfacing current $\bar{I}_{\mathrm{s}}$ and voltage $\bar{U}_{\mathrm{s}}$ it is required to determine the mathematical dependencies $P_{u}=F_{u}\left[\bar{U}_{\mathrm{s}}, \bar{I}_{\mathrm{s}}\right]$ and $P_{v p}=F_{v p}\left[\bar{U}_{\mathrm{s}}\right.$, $\left.\bar{I}_{\mathrm{s}}\right]$. The functions $F_{u}$ and $F_{v p}$ refer to the specific current source and specific installation as well as to the specific setting up of the surfacing method and conditions. To obtain these functions it is necessary to gather the experimental database for each of the methods, types and modes of surfacing, for which the installation is intended.

To obtain these dependencies and filling up the computer database for the surfacing modes a series of experiments on surfacing using fluxcored wires with open arc, under flux and in shielding gases was carried out. The experiments were performed in universal surfacing installation U-653, equipped with rectifier VDU 506. It allows surfacing the plane parts and the parts such as rotation bodies.

The registration of surfacing current and voltage in these experiments was performed using the developed computer information-and-measuring system (CIMS). The digitalization and data entry of the primary converters into PC were performed using measuring voltage converter E14-140 of L-Card company with the builtin 14-discharge ADC. The registration of parameters was carried out at frequency of $1 \mathrm{kHz}$. The input data processing was performed using the specially developed software in the environment of Microsoft Visual Studio 2010. For analysis of the output system data the standard software was used, such as PowerGraph, MATLAB, etc. According to the data entered into the computer the current state of the investigated process (arc ignition, arc breaks, short circuits of arc gap, arc burning periods) is identified automatically and the current and voltage parameters are calculated for the corresponding surfacing process.

In general, CIMS provides control and registration of the following surfacing process parameters: current values of surfacing voltage $U_{\mathrm{s}}(t)$, current values of surfacing current $I_{\mathrm{s}}(t)$, current values of arc voltage $U_{\mathrm{a}}(t)$ and $\operatorname{arc}$ current $I_{\mathrm{a}}(t)$; $U_{\mathrm{a}}(t)$ and $I_{\mathrm{a}}(t)$ at that were determined by excluding the voltage and current values from $U_{\mathrm{s}}(t)$ and $I_{\mathrm{s}}(t)$ in the time intervals of short circuits and arc breaks; average arc voltage $\bar{U}_{\mathrm{s}}$ and arc current $\bar{I}_{\mathrm{s}}$ during the surfacing period; entry of specifications for arc voltage $U_{\text {a.sp }}$ and arc current $\bar{I}_{\text {a.sp }}$; manual entry and indication of $P_{u \text { sp }}$ and $P_{v p \text { sp }}$ values; indication of working area of the approximating functions (in $\bar{U}_{\mathrm{s}}$ and $\bar{I}_{\mathrm{s}}$ parameters), providing the accuracy of approximation of voltage $\pm 1 \mathrm{~V}$ and \pm 10 A of current.

The main CIMS interface is shown in Figure 1, $a$. According to the results of surfacing the protocol of surfacing is formed (Figure 1, b). To the protocol all the necessary information is entered, which is later used for analysis, processing and formation of approximating functions.

To carry out the above mentioned experimental investigations 9 pilot batches of flux-cored wires of 1.8, 2.4 and $2.8 \mathrm{~mm}$ diameter were manufactured for surfacing under flux, with open arc and in shielding gases. The metal part of fluxcored wires charge was calculated in a way to provide producing of the deposited metal of the same type $-25 \mathrm{Kh} 5 \mathrm{FMS}$.

As the base metal the plates of steel St3 of $15 \mathrm{~mm}$ thickness were used. The pilot wires were used to perform surfacing of specimens in a wide range of surfacing conditions as to current and voltage. In the selected range of conditions the correlations between the position of potentiometers $P_{v p}$ and $P_{u}$, and the average values of arc current $I_{\mathrm{a}}$ and arc voltage $\bar{U}_{\mathrm{a}}$ were fixed, recorded by CIMS (see Figure 1).

In total, 9 series of experiments were carried out in the following ranges of surfacing conditions. In all the experiments the surfacing was performed at the same speed $-20 \mathrm{~m} / \mathrm{h}$.

- 19 experiments on open-arc surfacing using flux-cored wire of $1.8 \mathrm{~mm}$ diameter at current 160-260 A and voltage 22-29 V;

- 22 experiments on open-arc surfacing using flux-cored wire of $2.4 \mathrm{~mm}$ diameter at current 180-300 A and voltage 24-32 V;

- 34 experiments on open-arc surfacing using flux-cored wire of $2.8 \mathrm{~mm}$ diameter at current 200-300 A and voltage 24-32 V;

- 17 experiments on surfacing under flux AN26 using flux-cored wire of $1.8 \mathrm{~mm}$ diameter at current 240-300 A and voltage 26-31 V; 


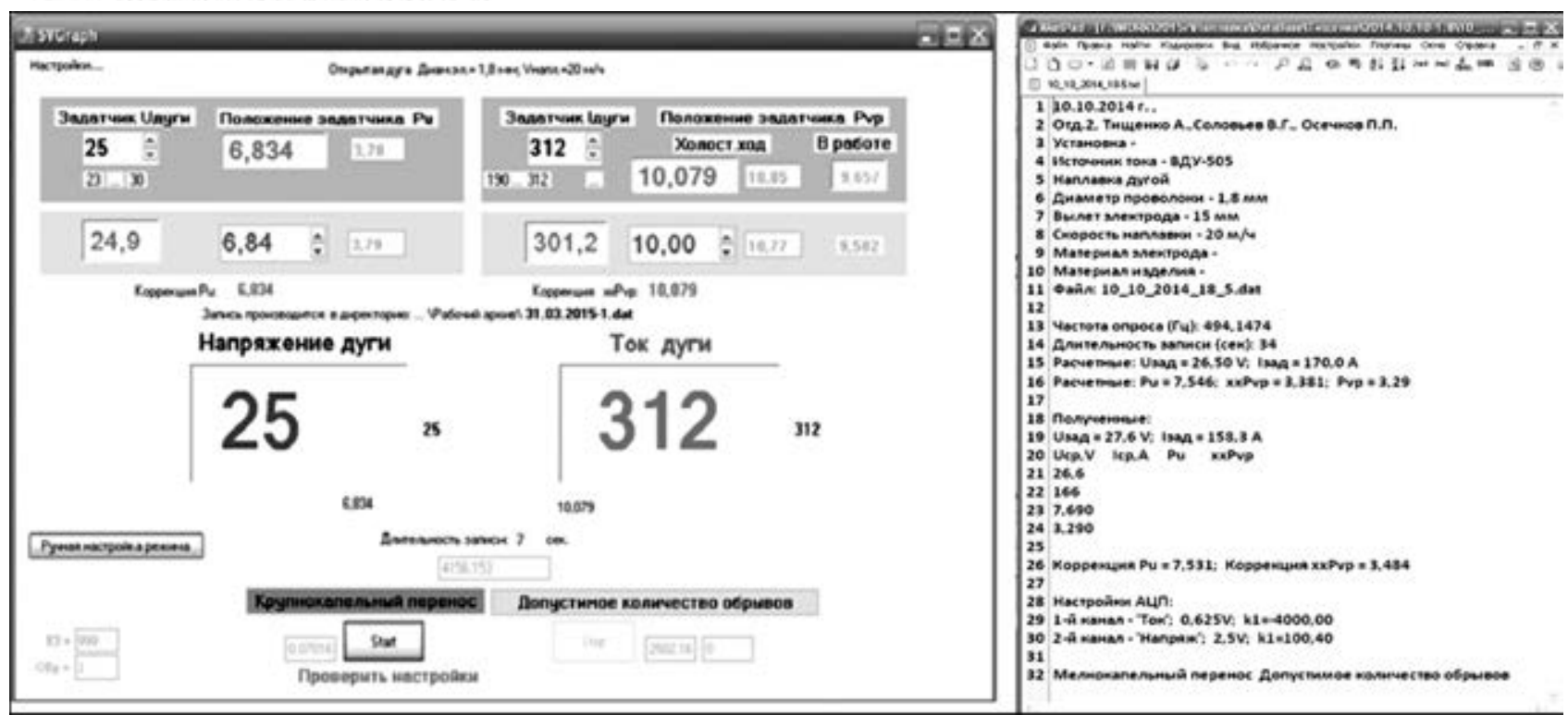

Figure 1. Basic interface of system for entry, monitoring and recording of surfacing parameters $(a)$, and protocol of recording the results of experiment on surfacing $(b)$

Results of experiments on open-arc surfacing using self-shielding flux-cored wire PP-Np-25Kh5FMS of 1.8 mm diameter showing the dependencies between position of potentiometers of voltage controller $P_{u}$ and controller of wire feed rate $P_{v p}$, and average values of voltage $\bar{U}_{\mathrm{a}}^{*}$ and current $\bar{I}_{\mathrm{a}}^{*}$ recorded by the system

\begin{tabular}{||c|c|c|c|c|c|c|c|c|c|}
\hline \hline $\begin{array}{c}\text { Number of } \\
\text { experiment }\end{array}$ & $\bar{U}_{\mathrm{a}}, \mathrm{V}$ & $\bar{I}_{\mathrm{a}}, \mathrm{A}$ & $P_{u}$ & $P_{v p}$ & $\begin{array}{c}\text { Number of } \\
\text { experiment }\end{array}$ & $\bar{U}_{\mathrm{a}}, \mathrm{V}$ & $\bar{I}_{\mathrm{a}}, \mathrm{A}$ & $P_{u}$ & $P_{v p}$ \\
\hline 1 & 27.6 & 179 & 7.69 & 3.29 & 14 & 23.8 & 193 & 6.42 & 5.01 \\
\hline 2 & 25.6 & 182 & 7.26 & 3.49 & 15 & 23.4 & 177 & 6.01 & 5.52 \\
\hline 3 & 26.5 & 206 & 7.54 & 3.38 & 16 & 26.5 & 251 & 7.77 & 5.49 \\
\hline 4 & 27.6 & 177 & 7.69 & 3.41 & 17 & 25.7 & 144 & 7.01 & 3 \\
\hline 5 & 25.2 & 233 & 7.41 & 4.27 & 18 & 23.4 & 156 & 5.61 & 5.03 \\
\hline 6 & 25.8 & 210 & 7.41 & 4.27 & 19 & 23.7 & 158 & 6.32 & 3.74 \\
\hline 7 & 27.8 & 211 & 7.91 & 4.23 & 20 & 25.0 & 160 & 6.95 & 3.74 \\
\hline 8 & 25.9 & 285 & 7.77 & 5.81 & 21 & 26.1 & 214 & 7.51 & 4.51 \\
\hline 9 & 22.7 & 265 & 6.92 & 6.14 & 22 & 23.9 & 174 & 6.33 & 3.90 \\
\hline 10 & 24.0 & 240 & 7 & 4.76 & 23 & 28.8 & 217 & 8.16 & 4.39 \\
\hline 11 & 23.1 & 197 & 6.44 & 4.76 & 24 & 23.7 & 200 & 6.82 & 5.16 \\
\hline 12 & 24.9 & 256 & 7.41 & 5.49 & 25 & 22.8 & 219 & 6.33 & 5.61 \\
\hline 13 & 24.0 & 176 & 6.42 & 4.53 & 26 & 24.0 & 285 & 7.32 & 5.61 \\
\hline
\end{tabular}

${ }^{*} \bar{U}_{\mathrm{a}}, \bar{I}_{\mathrm{a}}-$ values of arc voltage $U_{\mathrm{a}}(t)$ and $\operatorname{arc}$ current $I_{\mathrm{a}}(t)$, respectively, averaged during the period of surfacing.

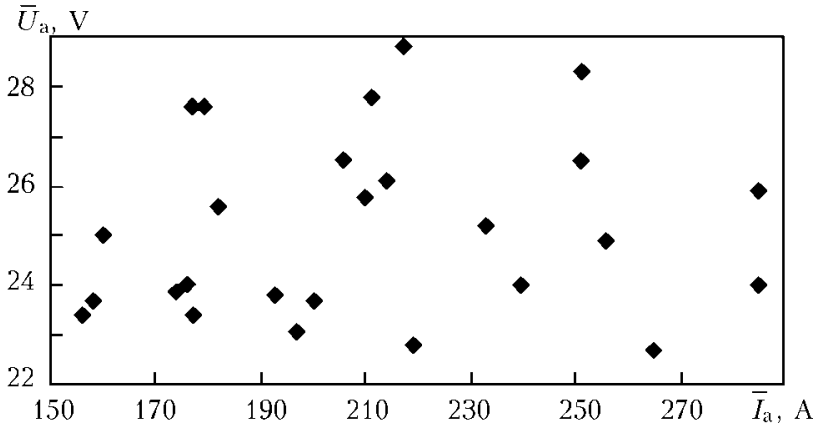

Figure 2. Area of used conditions during the experiments on open-arc surfacing using $1.8 \mathrm{~mm}$ flux-cored wire
- 25 experiments on surfacing under flux AN26 using flux-cored wire of $2.4 \mathrm{~mm}$ diameter at current 220-400 A and voltage 22-32 V;

- 24 experiments on surfacing under flux AN26 using flux-cored wire of $2.8 \mathrm{~mm}$ diameter at current 240-420 A and voltage 22-38 V;

- 14 experiments on surfacing in the mixture of shielding gases $\left(\mathrm{Ar}+18 \% \mathrm{CO}_{2}\right)$ using fluxcored wire of $1.8 \mathrm{~mm}$ diameter at current 220$310 \mathrm{~A}$ and voltage $20-30 \mathrm{~V}$;

- 12 experiments on surfacing in the mixture of shielding gases $\left(\mathrm{Ar}+18 \% \mathrm{CO}_{2}\right)$ using flux- 

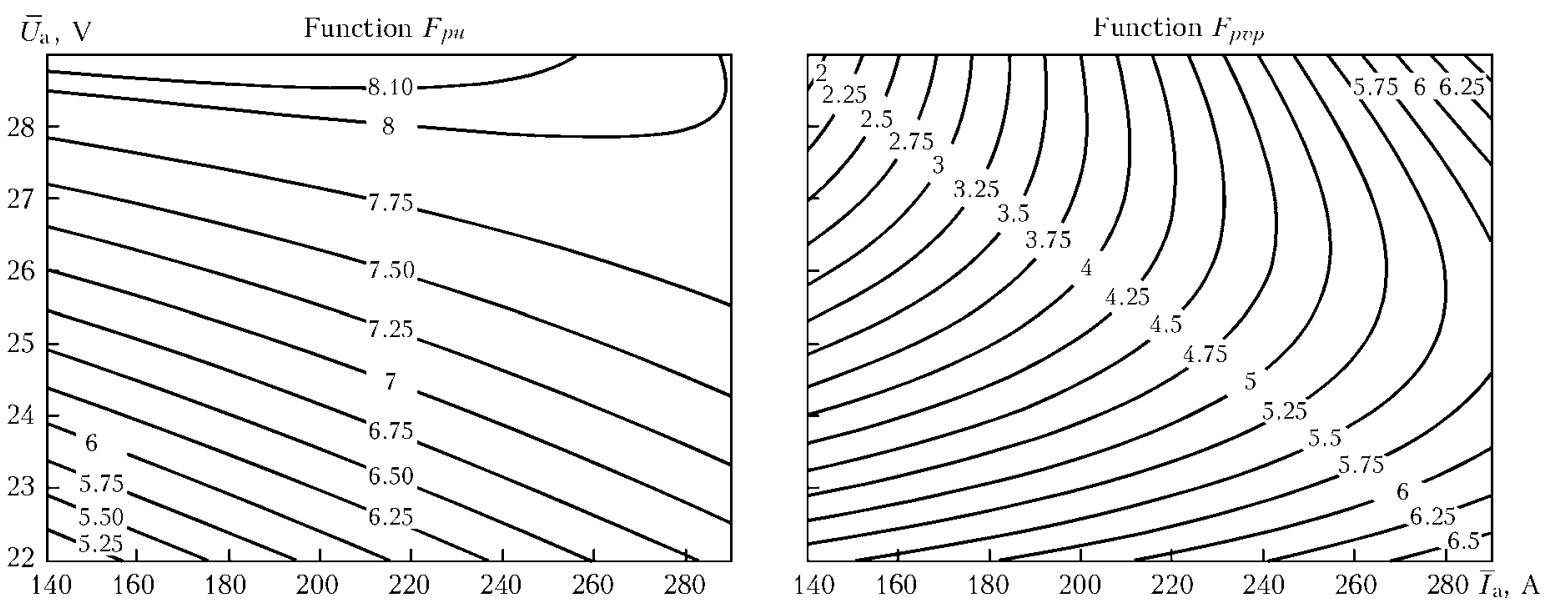

Figure 3. Diagrams of $F_{p u}$ and $F_{p v p}$ functions depending on average values of current and voltage in open-arc surfacing using self-shielding flux-cored wire of $1.8 \mathrm{~mm}$ diameter

cored wire of $2.4 \mathrm{~mm}$ diameter at current 210 $440 \mathrm{~A}$ and voltage $22-40 \mathrm{~V}$;

- 26 experiments on surfacing in the mixture of shielding gases $\left(\mathrm{Ar}+18 \% \mathrm{CO}_{2}\right)$ using fluxcored wire of $2.8 \mathrm{~mm}$ diameter at current 180 $340 \mathrm{~A}$ and voltage $22-40 \mathrm{~V}$.

As an example in the Table the results of experiments are given obtained in open-arc surfacing using self-shielding flux-cored wire of $1.8 \mathrm{~mm}$ diameter, and in Figure 2 the area of used surfacing conditions is shown.

The regression analysis of the data given in the Table and in Figure 2 was carried out, basing on its results the diagrams of functions $F_{p u}$ and $F_{p v p}$ were plotted depending on the average values of arc current and voltage in open-arc surfacing using flux-cored wire of $1.8 \mathrm{~mm}$ diameter (Figure 3).

Figure 4 shows the area of conditions of openarc surfacing using flux-cored wire of $1.8 \mathrm{~mm}$ diameter in coordinates $\bar{I}_{\mathrm{a}} / \bar{U}_{\mathrm{a}}$, wherein the approximation error during presetting the surfacing voltage and current does not exceed $\pm 1 \mathrm{~V}$ and $\pm 10 \mathrm{~A}$, respectively.

As a result of all 9 series of experiments the similar dependencies between the position of corresponding potentiometers for adjustment of voltage and wire feed speed and calculated average values of arc current and arc voltage were obtained, recorded by CIMS in the process of surfacing of each of approximately 200 deposited beads.

\section{Conclusions}

1. On the basis of portable computer and advanced software the information-and-measuring system was developed intended to investigate the peculiarities of arc surfacing. CIMS with accumulation of databases on technologies of arc surfacing of different parts can serve as a basis for creation of systems of automatic control of arc surfacing processes.

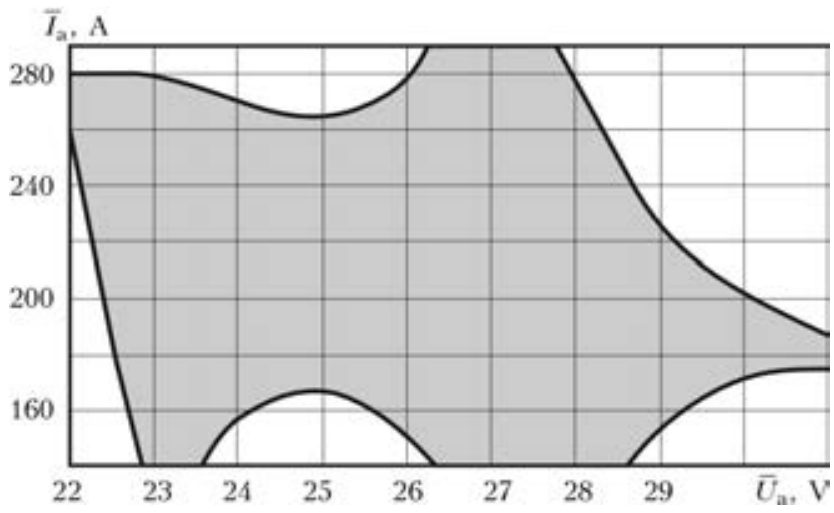

Figure 4. Range of open-arc surfacing modes with flux-cored wire of $1.8 \mathrm{~mm}$ diameter, where the approximation error in arc voltage and current during surfacing does not exceed $\pm 1 \mathrm{~V}$ and $\pm 10 \mathrm{~A}$, respectively

2. According to the results of experimental investigations on surfacing using flux-cored wires, with open arc, in shielding gases and under flux, the dependencies between the position of the corresponding potentiometers for adjustment of voltage and wire feed speed and average values of arc current and voltage for surfacing were determined, preset by the surfacing technologist. This allows using the developed CIMS with a high degree of accuracy to establish the surfacing modes by arc current and voltage without surfacing of reference specimens.

1. Bocharnikov, I.V., Gladky, P.V., Demchenko, V.F. et al. (1992) Expert system «Surfacing». In: Deposited metal. Composition, structure, properties, 9497. Kiev: PWI.

2. Demchenko, V.F., Kozlitina, S.S., Ryabtsev, I.A. (1998) Computer system of design of arc surfacing technology. Avtomatich. Svarka, 11, 61-66.

3. Lankin, Yu.N., Ryabtsev, I.A., Soloviov, V.G. et al. (2014) Effect of electric parameters of arc surfacing using flux-cored wire on process stability and base metal penetration. The Paton Welding J., 9, 25-29. 